\title{
Laser-assisted charge exchange as an atomic yardstick for proton beam energy measurement and phase probe calibration
}

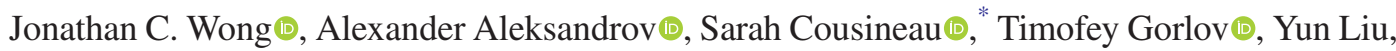 \\ Abdurahim Rakhman $\odot$, and Andrei Shishlo $\odot$ \\ Oak Ridge National Laboratory, Oak Ridge, Tennessee 37831, USA
}

(Received 30 September 2020; accepted 21 January 2021; published 11 March 2021)

\begin{abstract}
Laser-assisted charge exchange (LACE) is a novel scheme for injecting $H^{-}$ions into proton rings. Lorentz stripping of $H^{-}$ions into bare protons in magnetic dipoles is enabled by laser excitation that reduces the electron binding energy. We show that such atomic transitions impose very precise requirements on ion and laser parameters which can be utilized to measure the ion velocity. At the LACE experimental station at the Spallation Neutron Source (SNS), proton beam energy has been measured via LACE to a precision of $<1 \mathrm{MeV}$ for $\mathrm{a} \approx 1 \mathrm{GeV}$ beam. To verify the results against the existing energy measurement method at the SNS which calculates revolution frequency in the accumulator ring, we discuss how knowledge of the beam energy can be employed to synchronize phase probes. Such beam-based calibration using energy measurements via both LACE and the SNS ring show that the two methods produce consistent results.
\end{abstract}

DOI: 10.1103/PhysRevAccelBeams.24.032801

\section{INTRODUCTION}

\section{A. Ion beam energy measurement}

Accurate knowledge of the beam energy is crucial to the operation of ion accelerators. The time-of-flight (TOF) technique is the most commonly used method for measuring beam energy. Another, more exotic, example of energy measurement is laser spectroscopy, which was applied to $\mathrm{H}^{-}$beam in the early days of the LAMPF linac at LANL. Both techniques are briefly reviewed below.

TOF measurements require a beam line section with no accelerating field. Then beam-induced signals along phase probes at different locations constitute a linear arrival time versus distance relation that gives the beam velocity. This TOF technique is widely used in ion linacs for direct beam energy measurements [1-5]. Many rf tuning schemes for linacs [6-9] also employ variants of the TOF method that concern the change in beam energy rather than its absolute value.

Laser spectroscopy relies on the fact that hydrogen atoms and negative ions in a beam can be resonantly excited using a laser. Since an excitation only occurs if the beam energy corresponds to the given laser frequency and intersection angle, excitation signals can be observed to determine the

\footnotetext{
*Also at University of Tennessee.

Published by the American Physical Society under the terms of the Creative Commons Attribution 4.0 International license. Further distribution of this work must maintain attribution to the author(s) and the published article's title, journal citation, and DOI.
}

beam energy. A dedicated laser spectroscopy system was constructed at the LAMPF linac at LANL to determine the beam energy and momentum spread by scanning the laser frequency and collecting signals of neutral hydrogen atoms from autoionization of excited states [10,11].

In this study, we discuss how the system for laser-assisted charge exchange (LACE), a novel injection scheme into proton rings that makes use of lasers, can be directly employed to perform beam energy measurements. Such an application enhances the benefit of adopting LACE and also eliminates the need to construct a single-purpose laser system, as was done at LAMPF, for spectroscopy-based energy measurements. Since hydrogen atoms undergo negligible velocity change as LACE inverts their polarity, both the $H^{-}$beam energy upstream and the proton beam energy downstream are measured by LACE simultaneously.

\section{B. Beam-based phase probe calibration}

Regardless of whether there are other methods to measure the beam energy, ion accelerators typically contain phase probes which can be used for TOF energy measurements. At the Spallation Neutron Source, beam position monitors (BPMs) fulfill the role of phase pick-ups. To measure the absolute beam energy using TOF, phase probes must be calibrated such that phase shifts between probes correctly reflect flight time and do not contain systematic errors such as differences in cable lengths and electronics.

Phase calibration can be performed using a beam with known energy. Given the beam velocity, the phase advance between different probes can be calculated. Irrespective of their causes, the phase offsets can be measured as 
deviations from the calculated phase values and applied as corrections to future measurements.

\section{Spallation Neutron Source}

The Spallation Neutron Source (SNS) [12] delivers a $1 \mathrm{GeV}, 1.4 \mathrm{MW}$ proton driver beam for short pulse neutron production. The front end of the SNS accelerator complex consists of a $65 \mathrm{keV} \mathrm{H} H^{-}$source that produces a $1 \mathrm{~ms}$ beam at $60 \mathrm{~Hz}$, a radio-frequency quadrupole (RFQ), and a medium energy beam transport (MEBT) with chopper. The beam then undergoes successive acceleration from $2.5 \mathrm{MeV}$ to $87 \mathrm{MeV}$ in the drift tube linac (DTL), $186 \mathrm{MeV}$ in the coupled-cavity linac (CCL), and $1 \mathrm{GeV}$ in the superconducting linac (SCL).

The high energy beam transport (HEBT) transports the beam from the SCL to the accumulator ring where the $1 \mathrm{~ms}$ macro-pulse is compressed by accumulating its $645 \mathrm{~ns}$ mini-pulses over 1060 turns in the ring. After the entire pulse has been compressed to less than $1 \mu \mathrm{s}$, the beam is extracted and delivered via the ring to target beam transport (RTBT) to a liquid mercury target.

\section{Organization}

Section II describes laser-assisted charge exchange (LACE) and how it can be used to measure proton beam energy. Time-of-flight in the accumulator ring, another method to measure the beam energy at the SNS, is explained in Sec. III. Section IV discusses how beam energy measurement results can be utilized to calibrate phase probes. The calibration enables future energy measurements using time-of-flight technique only, and provides a means to benchmark the energy measurement techniques in the two previous sections. Section V concludes the study and outlines further work.

\section{PROTON BEAM ENERGY MEASUREMENT VIA LASER-ASSISTED CHARGE EXCHANGE}

\section{A. Laser-assisted charge exchange (LACE)}

Charge exchange injection is a common technique for injecting protons into rings. At the injection point, $\mathrm{H}^{-}$ions from a linac are stripped of their electrons as they merge with circulating protons in a dipole. Since charge exchange is a non-Liouvillian process, this technique enables an increase in the phase space density in the ring.

Stripping foils are commonly inserted to induce the charge exchange process. Foils have limited lifetime due to degradation and must avoid exceeding their temperature limit during operation. Furthermore, scattering in the foil often constitutes the main source of beam loss. All these limitations of stripping foils exacerbate with increasing beam intensity, which motivates efforts to look for other stripping schemes.

Laser-assisted charge exchange (LACE) is a proposed stripping technique at injection [13] to replace foil-based schemes presently in use. A schematic of the SNS LACE is shown in Fig. 1. Full stripping of $H^{-}$takes place in a threestage process: (1) Lorentz stripping of the outer electron in a dipole; (2) excitation of the hydrogen atom by a laser; and (3) Lorentz stripping of the excited electron. The second stage of LACE is a resonance process which can be utilized to measure the energy of the beam.

\section{B. SNS LACE experiment: LACE energy and its uncertainties}

There is an ongoing experimental program at the SNS to study LACE $[14,15]$. The experimental station has a $354.8 \mathrm{~nm}$ laser which crosses the beam line at a $37.5^{\circ}$ angle. The system is designed to excite electrons from the $n=1$ state to the $n=3$ state.

The LACE energy, i.e., the nominal beam energy at which the excitation process should occur, is given by:

$$
\gamma(1+\beta \cos \alpha)=\frac{\lambda}{\lambda_{0}}
$$

where $\gamma$ is the Lorentz factor, $\lambda_{0}$ is the $n=3$ transition wavelength in the Lyman series, and $\lambda$ is the laser wavelength. $\alpha$, the crossing angle between the laser and the beam, can be further decomposed into three parts:

$$
\alpha=\alpha_{0}+x_{0}^{\prime}+\delta D^{\prime}
$$

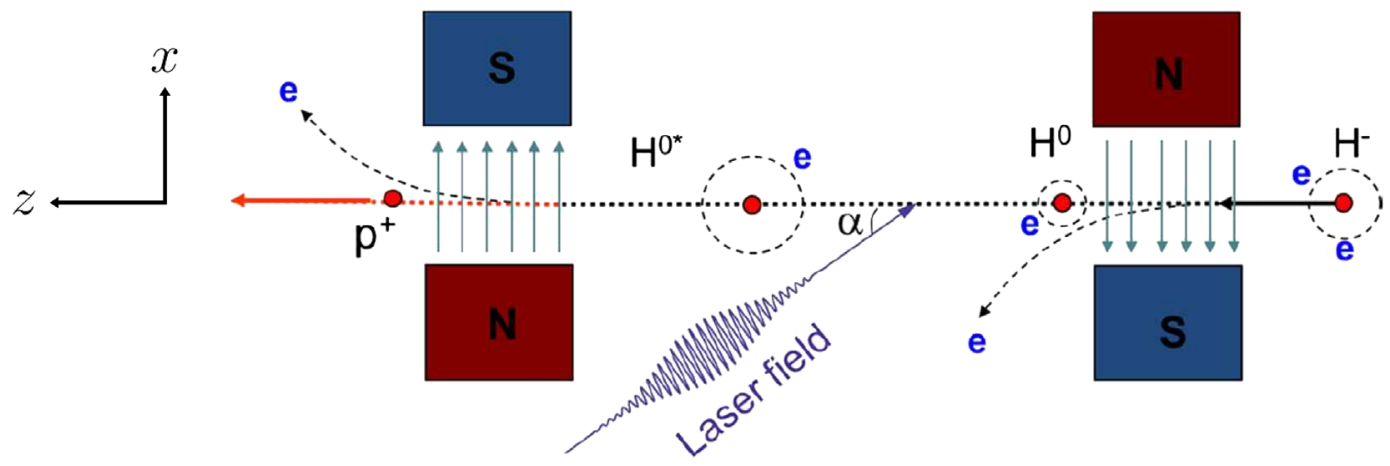

FIG. 1. Schematic of laser-assisted charge exchange (LACE). Figure reproduced from [14] with modifications. 
where $\alpha_{0}$ is the intersection angle between the laser and the reference trajectory, $x_{0}^{\prime}$ is the centroid angle of the beam and $\delta D^{\prime}$ is the angle due to dispersion with:

$$
\delta \equiv \frac{p-p_{0}}{p_{0}}=\frac{\sqrt{\gamma^{2}-1} m_{H^{-}} c-p_{0}}{p_{0}}
$$

with $m_{H^{-}}$being the mass of $H^{-}$and $p_{0}$ being the reference momentum of the beam line. In the experiment, $\delta$ is zero by design because the reference energy of the beam line is set to equal the nominal LACE energy whose value will be shown in Eq. (9). The values and uncertainties of all the parameters listed above are given in Table I.

Standard error propagation analysis shows that the error in the Lorentz factor is given by

$$
\begin{aligned}
\sigma_{\gamma}^{2}=( & \left.\frac{\partial \gamma}{\partial \alpha_{0}}\right)^{2} \sigma_{\alpha_{0}}^{2}+\left(\frac{\partial \gamma}{\partial x_{0}^{\prime}}\right)^{2} \sigma_{x_{0}^{\prime}}^{2} \\
& +\left(\frac{\partial \gamma}{\partial D^{\prime}}\right)^{2} \sigma_{D^{\prime}}^{2}+\left(\frac{\partial \gamma}{\partial \lambda}\right)^{2} \sigma_{\lambda}^{2}
\end{aligned}
$$

where

$$
\begin{gathered}
\frac{\partial \gamma}{\partial \alpha_{0}}=\frac{\partial \gamma}{\partial x_{0}^{\prime}}=K \sqrt{\gamma^{2}-1} \sin \left(\alpha_{0}+x_{0}^{\prime}+\delta D^{\prime}\right) \\
\frac{\partial \gamma}{\partial D^{\prime}}=K \delta \sqrt{\gamma^{2}-1} \sin \left(\alpha_{0}+x_{0}^{\prime}+\delta D^{\prime}\right) \\
\frac{\partial \gamma}{\partial \lambda}=\frac{K}{\lambda_{0}}
\end{gathered}
$$

with

$$
K=\frac{1}{1+\frac{\gamma\left(\lambda / \lambda_{0}-\gamma\right)}{\gamma^{2}-1}-\frac{\gamma m c D^{\prime}}{p_{0}} \sin \left(\alpha_{0}+x_{0}^{\prime}+\delta D^{\prime}\right)} .
$$

Using the parameters from Table I, the designed LACE energy in the SNS experiment and its errors are:

$$
E_{\mathrm{LACE}}=(\gamma-1) m_{H^{-}} c^{2}=981.0 \pm 0.6 \mathrm{MeV} .
$$

\section{Energy measurement}

Since both the laser frequency and the intersection angle are kept constant in the experiment, instead of measuring the beam energy directly, the beam energy is varied until it equals a designated value, i.e., the nominal laser stripping energy.

To determine when the beam energy equals the LACE energy, the rf phase of the last acting cavity of the SNS linac was scanned over a range at $1^{\circ}$ steps such that the resulting beam energies should contain the nominal energy for laser stripping. Since only hydrogen atoms that are
TABLE I. Parameters relevant to the LACE energy in the SNS experiment.

\begin{tabular}{ll}
\hline \hline Parameter & \multicolumn{1}{c}{ Value } \\
\hline$\alpha_{0}$ & $37.5^{\circ} \pm 0.008^{\circ}$ \\
$\lambda_{0}$ & $102.5734 \mathrm{~nm}$ \\
$\lambda$ & $(1064.4 \pm 0.1 \mathrm{~nm}) / 3$ \\
$x_{0}^{\prime}$ & $0.0 \pm 1.0 \mathrm{mrad}$ \\
$D^{\prime}$ & $0.0 \pm 0.5 \mathrm{rad}$ \\
$m_{H^{-}}$ & $939.294 \mathrm{MeV} / c^{2}$ \\
\hline \hline
\end{tabular}

stripped into protons generate BPM signals, the amplitude of the BPM reading downstream of the stripping station (i.e., to the left of the setup in Fig. 1) is proportional to the stripping efficiency.

Apart from the beam energy, the variation of $\sigma_{y}$, the vertical beam size at the interaction point, with respect to cavity phase also affects the stripping efficiency. When the cavity phase varied from $-95.0^{\circ}$ to $-55.0^{\circ}$, a range that contained all nonvanishing signals, simulation results showed that $\sigma_{y}$ attained values between $0.33 \mathrm{~mm}$ and $0.40 \mathrm{~mm} . \sigma_{\gamma}$, the laser spot size at the interaction point, was $0.25 \mathrm{~mm}$. Following the treatment for luminosity in colliding beams, a model with aligned, overlapping Gaussian distributions of photons and $H^{-}$ions is employed to approximate the number of stripped ions per pulse by:

$$
N_{\text {stripped }}\left(E, \sigma_{y}\right)=\frac{C_{1} f(E)}{\sqrt{\sigma_{\gamma}^{2}+\sigma_{y}^{2}}}
$$

where $C_{1}$ is some constant and $f(E)$ describes how the stripping efficiency varies as a function of energy when all other parameters remain unchanged. Rearranging terms give:

$$
f(E) \propto N_{\text {stripped }}\left(E, \sigma_{y}\right) \sqrt{\sigma_{\gamma}^{2}+\sigma_{y}^{2}}
$$

which shows that, for each cavity phase $\phi$, the signal amplitude $I(\phi)$ can be corrected for the dependence on $\sigma_{y}$ by rescaling it as follows:

$$
I(\phi) \rightarrow I(\phi) \sqrt{\sigma_{\gamma}^{2}+\sigma_{y}(\phi)^{2}}
$$

Here we emphasize $\sigma_{y}(\phi)$ is a function of $\phi$, its value can be obtained from accelerator models. The corrected signals, which have the same functional form as $f(E)$ that only describes how the stripping efficiency varies with beam energy, are thus exactly the data needed for performing energy measurements.

A plot of the rf cavity phase versus the corrected amplitude of the left electrode on the BPM downstream of the stripping station is shown in Fig. 2. Gaussian curve fitting by the optimize module in SciPy [16] has found: 


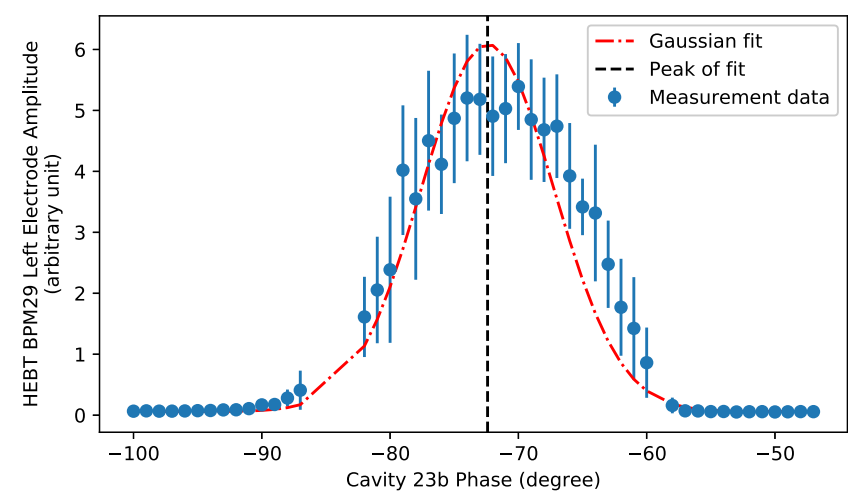

FIG. 2. Signal amplitude on the left electrode of the BPM downstream of the LACE study station versus rf phase of cavity 23b. Signal amplitudes are corrected using Eq. (12) to account for the effects of changing vertical beam size during the scan. Measurement data for cavity phases from $-86^{\circ}$ to $-83^{\circ}$ were lost due to data logging issues.

$$
\phi_{\text {peak }}=-72.4^{\circ} \pm 0.1^{\circ}
$$

to be the cavity phase at which the stripping efficiency is maximum. The other three electrodes on the BPM have different amplifications on their signals, they either saturated during the cavity phase scan or produced the same $\phi_{\text {peak }}$ as the left electrode did. Their measurements are not shown here.

To equate the cavity phase at which the stripping efficiency peaks with the cavity phase at which the beam has the nominal LACE energy given by Eq. (1), it is assumed that each of the following distributions is roughly symmetric about its mean: beam energy spread, beam horizontal angular spread, and laser divergence. In that case, the spreads only act to broaden the stripping efficiency vs cavity phase plot without changing the peak location. There is little effect on energy measurement apart from making the peak less pronounced and harder to locate.

\section{ENERGY MEASUREMENT USING THE SNS RING}

An alternative method to measure the beam energy at SNS is to count the beam's revolution frequency in the accumulator ring. Unfortunately, the beam whose energy was measured in Sec. II C's LACE experiment could not be sent directly into the ring because the ring was not configured for $981 \mathrm{MeV}$ protons and beam time limitations forbade extensive tuning of the ring. In this section, we present experimental results on beams with the routine energy during normal operations. The comparison between LACE-based and ring-based energy measurement methods is treated in detail in Sec. IV.

The path length of the beam in the ring per revolution is given by $L=248.009 \pm 0.010 \mathrm{~m}$. The circumference of the ring is $248.000 \mathrm{~m}$, whereas $L$ is roughly $0.009 \mathrm{~m}$ longer due to the trajectory in the injection region whose schematic layout is shown in Fig. 3. There is a further, smaller correction to the path length which arises from the strength of the kicker magnets-this effect is estimated via frequency measurements as explained below. The main source of uncertainty in $L$ arise from misalignments throughout the entire ring, and we estimate their total effect should be no larger than $0.01 \mathrm{~m}$.

To measure the beam energy, 1 minipulse was injected into the ring and stored for 1000 turns. The procedure was repeated 100 times and the revolution frequency was found to be: $f=(1.05973 \pm 0.00010) \times 10^{6} \mathrm{~Hz}$.

There are two sources of uncertainty in the revolution frequency. The first one concerns the additional path length in the injection region that depends on the strength of the dynamic kickers. Two measurements were performed at two typical kicker strengths and the revolution frequency was found to have varied by about $10 \mathrm{~Hz}$. The second, dominant uncertainty that amounts to $100 \mathrm{~Hz}$ arises from how the revolution frequency is determined from the peak of the discrete Fourier transform of the beam current monitor measurements.

The beam velocity was found from $v=L f$, where the associated beam energy is

$$
E_{\text {ring }}=1012.1 \pm 0.7 \mathrm{MeV} \text {. }
$$

\section{BEAM-BASED PHASE PROBE CALIBRATION AND TIME-OF-FLIGHT ENERGY MEASUREMENT}

This section discusses how energy measurement results can be used to calibrate phase probes. We refer to BPM phases below since BPMs fulfill the role of phase probes at

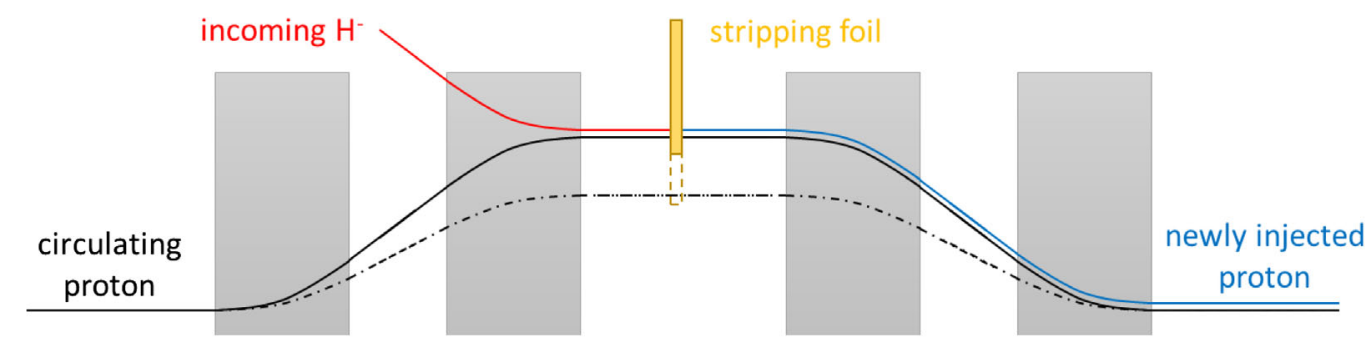

FIG. 3. Schematic of the injection region in the SNS ring. Combinations of dipoles and dynamic kickers are depicted in grey. Dotted lines denote the beam's path and position of the stripping foil when the magnets have a different strength. 
the SNS. The purposes of the discussion are two-fold. First, the calibration enables one to use BPMs for future time-offlight energy measurements, which is more convenient in operation than the two methods used above. Second, in the context of this study, beam-based BPM calibration can serve as a consistency check between the two energy measurement methods. As explained in Sec. III, the two methods were applied to measure beams with different energies. While these circumstances prevent direct benchmarking between the two methods, BPM calibration enables one to compare the results in a meaningful way.

\section{A. Beam-based phase calibration}

Once a beam's velocity is known, it can be used to calibrate the BPM phases. Ideally, the BPM phase measured by the $i$ th BPM is given by:

$$
\tilde{\varphi}_{i}=\varphi_{i}+\Delta \varphi_{i}
$$

where $\tilde{\varphi}$ is the measured phase of the BPM, $\varphi$ is the true phase and $\Delta \varphi$ is the phase offset relative to a reference that is set during the calibration process. All phases $\in$ $\left(-180^{\circ}, 180^{\circ}\right]$ by convention.

The reference for phase calibration consists of both a position, which we denote by $z_{0}$, and a phase at the position, which is chosen to be $0^{\circ}$. Then, given a beam with known velocity, the true phase at any BPM location is given by:

$$
\varphi_{i}=\left(\frac{z_{i}-z_{0}}{v} 360^{\circ} f\right)+360^{\circ} k_{i}
$$

where $z_{i}$ is the location of the $i$ th BPM, $f$ is the rf frequency of the phase probe system and $k_{i}$ is the integer number of $\mathrm{rf}$ periods shifted to render $\varphi_{i} \in\left(-180^{\circ}, 180^{\circ}\right]$.

During actual experiments, there are jitter in the timing, beam velocity and BPM phases. The measured phase of the $i$ th BPM during the $j$ th measurement can be written as:

$$
\begin{aligned}
\tilde{\varphi}_{i}^{(j)}= & \varphi_{0}+\Delta \varphi_{i}+\frac{\left(z_{i}-z_{0}\right)}{v} 360^{\circ} f+360^{\circ} k_{i} \\
& +\delta \varphi_{0}^{(j)}+\delta \varphi_{i}^{(j)}-\frac{\left(z_{i}-z_{0}\right)}{v^{2}} 360^{\circ} f \delta v^{(j)}
\end{aligned}
$$

where $\varphi_{0}$ is the phase at the reference position which is taken to be $0^{\circ}$ and $\delta \varphi_{0}^{(j)}$ is timing jitter. $\delta \varphi_{i}^{(j)}$ is the phase jitter of the $i$ th BPM and $\delta v^{(j)}$ is the velocity jitter of the beam.

Since all jitter terms have vanishing expectation values, averaging over a set of measurements and rearranging terms give:

$$
\Delta \varphi_{i}=\left\langle\tilde{\varphi}_{i}\right\rangle-\left(\frac{z_{i}-z_{0}}{v} 360^{\circ} f\right)+360^{\circ} k_{i}
$$

where $\left\langle\tilde{\varphi}_{i}\right\rangle$ is the mean of measured phases. The phase offset thus obtained can be saved and applied to future phase measurements. Further analysis of uncertainties is discussed in the Appendix.

\section{B. Experimental results}

To perform phase calibration with experiments on beam energy measurements, phases of the BPMs after the last accelerating cavity in the linac were recorded. For energy measurement using the SNS ring, the measured phases could be used directly. For energy measurement using LACE, the BPM phases were recorded throughout the cavity phase scan. Once the cavity phase corresponding to the nominal beam energy is known, the phase that each BPM would have at the nominal LACE energy can be obtained by linear interpolation.

Results of BPM phase calibration using the SNS ring and LACE are shown in Fig. 4. The phase offsets are calculated by Eq. (18) using knowledge of the beam velocity. There are more BPMs in the HEBT, but only the first four are included in the analysis because the ones further

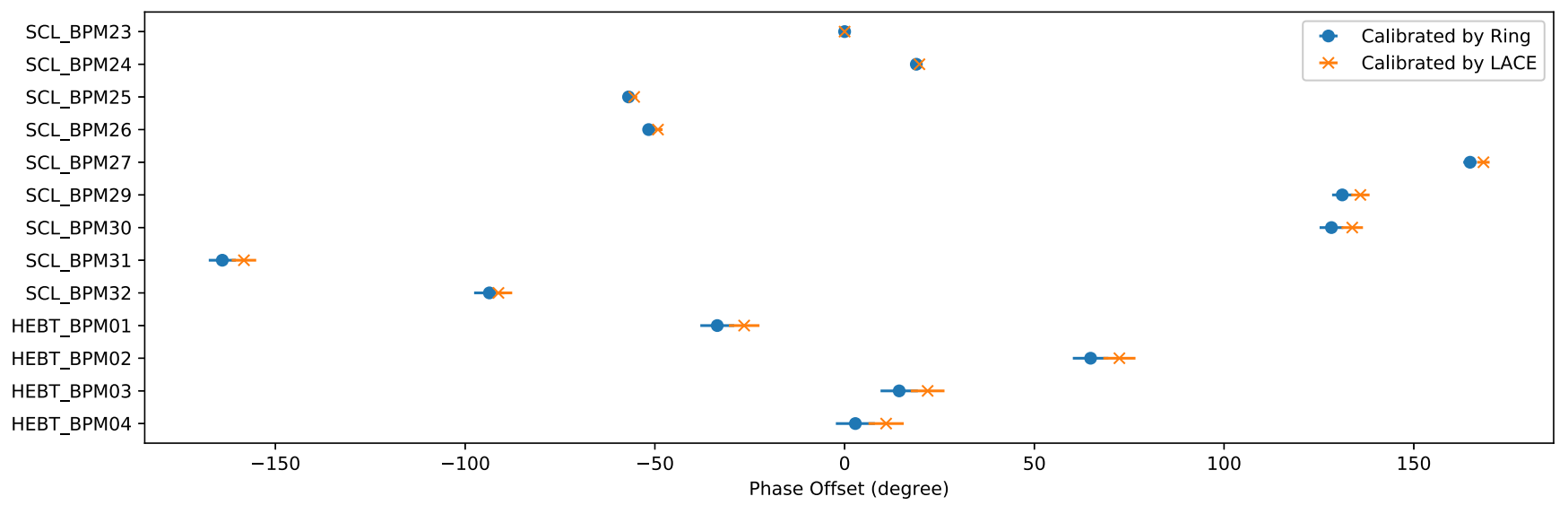

FIG. 4. Phase offsets, with error bars, of BPMs in the SNS SCL and HEBT from calibration experiments conducted in February (ring) and April (LACE) 2020. Each phase offset and its error are calculated using Eq. (18) and (A1) respectively. 
downstream have older designs that render them more susceptible to phase shifts over time.

Since the beam had different velocities during the ring experiment and the LACE experiment, it is meaningless to compare the measured BPM phases. The BPM phase offsets are inherent properties of the BPMs and the rf reference system, so their calibrated values from the two experiments can be compared. As shown in Fig. 4, the two sets of phase offsets agree roughly. However, two issues prevent phase offsets per se from being a clear consistency check on the two energy measurement methods. First, as a consequence of how uncertainties in the beam velocity propagate into uncertainties in the phase offsets [see Eq. (A1)], differences in calibrated phase offsets and their errors increase with distance from the reference point. Secondly, the phase offsets of every BPM can be compared, so it is unclear how to quantify the overall agreement from a collection of comparisons. To avoid both issues, we show in the next two subsections how using the calibrated phase offsets to perform TOF measurements constitute a direct and quantifiable consistency check between the two energy measurement methods.

\section{TOF Measurements with Beam-Calibrated BPMs}

Having obtained the phase offset for each BPM, subsequent TOF energy measurements using the phases of $n$ BPMs amount to finding the slope in the time-distance graph by solving:

$$
\frac{1}{360^{\circ} f}\left(\begin{array}{c}
\varphi_{1} \\
\varphi_{2} \\
\varphi_{3} \\
\vdots \\
\varphi_{n}
\end{array}\right)=\left(\begin{array}{cc}
z_{1}-z_{0} & 1 \\
z_{2}-z_{0} & 1 \\
z_{3}-z_{0} & 1 \\
\vdots & \vdots \\
z_{n}-z_{0} & 1
\end{array}\right)\left(\begin{array}{l}
a \\
b
\end{array}\right)
$$

where $z_{i}$ and $\varphi_{i}$ are the position and the offset-corrected phase of the $i$ th BPM respectively. $\varphi_{i}$ is given by:

$$
\varphi_{i}=\tilde{\varphi}_{i}-\Delta \varphi_{i}+360^{\circ} k_{i}
$$

with $\tilde{\varphi}_{i}$ and $\Delta \varphi_{i}$ being its measured phase and its phase offset from beam-based calibration respectively. Note that prior knowledge on the range of the beam energy is required to determine $k_{i}$, the integer number of rf periods between two arrival times.

The beam velocity to be obtained from the TOF measurements, $\tilde{v}$, is simply given by:

$$
\tilde{v}=a^{-1}
$$

whose errors are analyzed in detail in Appendix. $b$, the other quantity obtained by solving Eq. (19), is the beam's arrival time at the reference position $z_{0}$.

\section{Consistency check on energy measurement using LACE}

Having developed the treatment on phase calibration and TOF calculations, we have three methods to obtain the beam energy during each experiment: I. direct measurement; II. TOF calculations using ring-calibrated phase offsets; and III. TOF calculations using LACE-calibrated phase offsets. For Method I, uncertainties of the measured beam energies come from the respective sources discussed in Sec. II B and Sec. III. Methods II and III employ all 13 BPMs listed in Fig. 4 to perform TOF calculations of the beam velocity via $\mathrm{Eq}$ (19). Uncertainties in the beam energies from TOF calculations with multiple BPMs are obtained using the error analysis developed in Appendix. The results are displayed in Table II.

To check for consistency between the two energy measurement methods, ring and LACE, one can compare: (a) the beam energy measured directly by LACE; against (b) the beam energy calculated from TOF using ringcalibrated phase offsets, and vice versa. From Table II, we observe that the error bars for LACE energy (I) and LACE energy (II) overlap. The same is true for ring energy (I) and ring energy (III). These agreements between direct measurements and TOF calculations indicate that the two energy measurement methods produce consistent results.

One can also compare the measured energy versus TOF calculations using phase offsets calibrated by the same method. While agreement is guaranteed by the circular nature of the comparison, the uncertainty of the latter would be larger than that of the former due to contribution of phase jitter when one calculates the beam velocity via Eq. (19). As can be seen from LACE energy (I) versus LACE energy (III) and ring energy (I) versus ring energy

TABLE II. Method I denotes measured energy; Method II denotes energy from TOF calculations using ring-calibrated phase offsets; and Method III denotes energy from TOF calculations using LACE-calibrated phase offsets. LACE energy refers to the energy of $H^{-}$, and ring energy that of bare proton, during the respective experiments described in Sec. II and Sec. III. TOF velocities and their errors are calculated using Eq. (21) and (A12) respectively-conversion from velocities to energies is trivial.

\begin{tabular}{lccc}
\hline \hline Method & I & II & III \\
\hline LACE energy & $981.0 \pm 0.6 \mathrm{MeV}$ & $980.0 \pm 0.6 \mathrm{MeV}$ & $981.0 \pm 0.6 \mathrm{MeV}$ \\
Ring energy & $1012.1 \pm 0.7 \mathrm{MeV}$ & $1012.1 \pm 0.7 \mathrm{MeV}$ & $1013.1 \pm 0.6 \mathrm{MeV}$ \\
\hline \hline
\end{tabular}


(II), the differences in uncertainties are small. These results mean that uncertainty in the beam velocity during the calibration process is the dominant sources of uncertainty in subsequent TOF measurements.

\section{CONCLUSION}

LACE is a novel scheme for injecting $H^{-}$into proton rings with the help of lasers. We demonstrated that the setup for LACE can be applied to measure proton beam energy. This extra application enhances the benefits of adopting LACE and its success is testimony to the growing impact of lasers in accelerators. Detailed error analysis was performed to show that LACE can measure the energy to a precision of $<1 \mathrm{MeV}$ for a $\approx 1 \mathrm{GeV}$ beam. The energy measurement was applied to beam-based calibration of the BPM phases along the linac which would enable future TOF energy measurements. Energy measurement using LACE was shown to produce results that are consistent with energy measurement using the revolution frequency in the SNS ring.

Energy measurement by LACE should also work in proposed configurations of the LACE setup in the future [17] which use different excitations. In addition to exploring the implications of new LACE systems, further work includes studies to increase the sensitivity of the stripping efficiency against the beam energy (i.e., make the curve in Fig. 2 narrower). One possibility is modifications on the beam optics with emphasis on exploiting divergence. Improved sensitivity will enhance the precision of energy measurements via LACE and benefit all future TOF measurements using phase probes thus calibrated.

\section{ACKNOWLEDGMENTS}

We are grateful to Jeffrey Holmes and Willem Blokland from Oak Ridge National Laboratory for their support on calculations of the beam's path length and revolution frequency in the SNS accumulator ring respectively. We thank Martin Kay from University of Tennessee for providing the errors on the laser angle in the SNS LACE experiment. This research used resources of the Spallation Neutron Source, which is a DOE Office of Science User Facility. This manuscript has been authored by UT-Battelle, LLC, under Contract No. DE-AC05-00OR22725 with the U.S. Department of Energy (DOE). The U.S. government retains and the publisher, by accepting the article for publication, acknowledges that the U.S. government retains a nonexclusive, paid-up, irrevocable, worldwide license to publish or reproduce the published form of this manuscript, or allow others to do so, for U.S. government purposes. DOE will provide public access to these results of federally sponsored research in accordance with the DOE Public Access Plan [18].

\section{APPENDIX: ERROR ANALYSIS OF TOF MEASUREMENTS WITH BEAM-CALIBRATED PHASE PROBES}

This Appendix provides a detailed treatment on the effects of beam-based calibration on phase offset calculations and the associated errors in TOF energy measurements.

It is important to distinguish between two beam velocities. We denote by $v$ the velocity of the beam during the calibration process, which is known. The unknown beam velocity that is to be found from TOF measurements is denoted by $\tilde{v}$.

From Eq. (18), errors in the phase offsets are given by:

$$
\sigma_{\Delta \varphi_{i}}^{2}=\sigma_{\tilde{\varphi}_{i}}^{2}+\left(\frac{z_{i}-z_{0}}{v^{2}} 360^{\circ} f\right)^{2} \sigma_{v}^{2}
$$

where $\sigma_{v}$ is the uncertainty in the beam velocity during the calibration process.

$\sigma_{\tilde{\varphi}_{i}}^{2}$, the standard deviation of the measured BPM phases, actually has contribution from all three jitter terms in Eq. (17). An analysis on the longitudinal jitter of the output beam from the SCL has found that: (i) the velocity jitter $\delta v^{(j)} \leq 7 \times 10^{-5} c$, which corresponds to an energy jitter $\leq 60 \mathrm{keV}$ for a $\approx 1 \mathrm{GeV}$ beam; (ii) the timing jitter $\delta \varphi_{0}^{(j)} \leq 0.1^{\circ}$; (iii) the phase jitter of the $i$-th BPM $\delta \varphi_{i}^{(j)} \leq$ $0.3^{\circ}$ for all $i$. Since the velocity jitter is an order of magnitude smaller than the uncertainty in the beam velocity, and the timing jitter is smaller than the phase jitter, we take $\sigma_{\tilde{\varphi}_{i}}^{2}$ to be the BPM phase jitter (i.e., noise) for simplicity.

Conventional least square is not the proper method to solve Eq. (19) because the errors in different $\varphi_{i}$ 's are correlated due to uncertainties in the beam velocity during calibration. The phase used for TOF measurements has three sources of uncertainties:

$$
\delta \varphi_{i}=\delta \tilde{\varphi}_{i}+\delta \tilde{\varphi}_{i}^{*}-360^{\circ} f \frac{\left(z_{i}-z_{0}\right)}{v^{2}} \delta v
$$

where $\delta \tilde{\varphi}_{i}$ is the error of the measured phase during TOF measurements, $\delta \tilde{\varphi}_{i}^{*}$ is the error of the measured phase during the calibration process, and $\delta v$ is uncertainty in the beam velocity during the calibration process. While the first two terms are uncorrelated among different BPMs, the third term is not:

$$
\sigma_{\varphi_{i} \varphi_{j}}=\frac{\left(z_{i}-z_{0}\right)\left(z_{j}-z_{0}\right)}{v^{4}}\left(360^{\circ} f\right)^{2} \sigma_{v}^{2}
$$

One way to resolve the problem is to make a linear transformation such that all off-diagonal covariance terms vanish in the new coordinates. Such a generalized least square method is, however, tedious and devoid of insight. 
Instead, we define new coordinates and slope:

$$
\begin{gathered}
\hat{\varphi}_{i} \equiv \varphi_{i}+\left(\frac{z_{i}-z_{0}}{v} 360^{\circ} f\right) \\
\hat{a} \equiv a+\frac{1}{v}
\end{gathered}
$$

such that the system of equations becomes:

$$
\frac{1}{360^{\circ} f}\left(\begin{array}{c}
\hat{\varphi}_{1} \\
\hat{\varphi}_{2} \\
\hat{\varphi}_{3} \\
\vdots \\
\hat{\varphi}_{n}
\end{array}\right)=\left(\begin{array}{cc}
z_{1}-z_{0} & 1 \\
z_{2}-z_{0} & 1 \\
z_{3}-z_{0} & 1 \\
\vdots & \vdots \\
z_{n}-z_{0} & 1
\end{array}\right)\left(\begin{array}{l}
\hat{a} \\
b
\end{array}\right) .
$$

The error in $\hat{\varphi}_{i}$ is given by:

$$
\delta \hat{\varphi}_{i}=\delta \tilde{\varphi}_{i}+\delta \tilde{\varphi}_{i}^{*}
$$

where both terms on the right-hand side are measurement noises and so there is no correlation between $\hat{\varphi}_{i}$ and $\hat{\varphi}_{i}$ for $i \neq j$. Therefore:

$$
\begin{gathered}
\sigma_{\hat{\varphi}_{i}}^{2}=\sigma_{\tilde{\varphi}_{i}}^{2}+\sigma_{\tilde{\varphi}_{i}^{*}}^{2} \\
\sigma_{\hat{\varphi}_{i}, \hat{\varphi}_{j}}=0 \text { for } i \neq j .
\end{gathered}
$$

Note that there are contributions to $\sigma_{\hat{\varphi}_{i}}^{2}$ from both $\sigma_{\tilde{\varphi}_{i}}^{2}$, the variation of the mean of the measured phase during TOF experiment, and $\sigma_{\tilde{\varphi}_{i}^{*}}^{2}$, the variation of the mean of the measured phase during the calibration process. They are not necessarily the same, both because the magnitude of the noise may vary and because the variation of the mean goes down with $1 / \sqrt{n}$ depending on the number of signals measured.

Solving Eq. (A6) by simple least square yields $\hat{a}$ and its variance $\sigma_{\hat{a}}$ from which one obtains:

$$
a=\hat{a}-\frac{1}{v}
$$

and

$$
\sigma_{a}^{2}=\sigma_{\hat{a}}^{2}+\left(\frac{\sigma_{v}}{v^{2}}\right)^{2}
$$

The beam velocity from TOF measurements $\tilde{v}$ is given by Eq. (21). Thus its uncertainty:

$$
\sigma_{\tilde{v}}^{2}=\frac{\sigma_{a}^{2}}{a^{4}}
$$

can be obtained from the results of Eq. (A10) and (A11).

Equation (A11) shows that the uncertainty in $\mathrm{TOF}$ energy measurements has contributions from two terms, which correspond to measurement noise and uncertainties in the beam velocity during calibration respectively.

This result above also agrees with the physical picture where the errors in TOF measurements should not depend on how we pick $z_{0}$. Although the choice of $z_{0}$ can make the variance in the phase offset arbitrarily large [see Eq. (A1)], the treatment above confirms the component of the error that comes from the choice of $z_{0}$ will not propagate into errors in TOF measurements.

[1] M. B. Popovic, T. L. Owens, T. K. Kroc, L. J. Allen, and C. W. Schmidt, Time-of-flight measurements of absolute beam energy in the fermilab linac, in Proceedings of the 15th Particle Accelerator Conference, PAC-1993, Washington, DC, 1993 (IEEE, New York, 1993), Vol. 3, pp. 1689-1690.

[2] S. Lee, Y.-S. Cho, H. Kim, and H.-J. Kwon, Measurements of the Beam Energy and Beam Profile of $100 \mathrm{MeV}$ Proton Linac at KOMAC, in Proc. of International Particle Accelerator Conference (IPAC'16), Busan, Korea, May 8-13, 2016, International Particle Accelerator Conference No. 7 (JACoW, Geneva, Switzerland, 2016) pp. 217-219.

[3] F. Galizzi, M. Caldara, and A. Jef, A time-of-flight based energy measurement system for the LIGHT medical accelerator, J. Phys. Conf. Ser. 1067, 072019 (2018).

[4] Y. Batygin, F. Shelley, and H. Watkins, Time-of-Flight, Beam-Energy Measurement of the LANSCE $805-\mathrm{MHz}$ Linac, in Proc. 9th International Particle Accelerator Conference (IPAC'18), Vancouver, BC, Canada, April 29-May 4, 2018, International Particle Accelerator Conference No. 9 (JACoW Publishing, Geneva, Switzerland, 2018), pp. 1075-1077.

[5] P. N. Ostroumov, S. Cogan, K. Fukushima, S. Lidia, T. Maruta, A. S. Plastun, J. Wei, J. Wong, T. Yoshimoto, and Q. Zhao, Heavy ion beam acceleration in the first three cryomodules at the facility for rare isotope beams at michigan state university, Phys. Rev. Accel. Beams 22, 040101 (2019).

[6] K. R. Crandall, delta. t tuneup procedure for the LAMPF 805-MHz linac, Los Alamos National Lab Tech. Report No. LA-6374-MS, 1976.

[7] T. L. Owens, M. B. Popovic, E. S. McCrory, C. W. Schmidt, and L. J. Allen, Phase scan signature matching for linac tuning, in Proceedings of the 17th International Linear Accelerator Conference (LINAC-1994), Tsukuba, Japan, 1994 (KEK, Tsukuba, Japan, 1994), Vol. 3, pp. 1691-1693.

[8] M. Ikegami, RF Tuning Schemes for J-PARC DTL and SDTL, in Proceedings of the 22nd International Linear Accelerator Conference (LINAC2004), Lübeck, Germany, 
16-20 August 2004 (JACoW, Geneva, Switzerland, 2005), pp. 414-416.

[9] J. Galambos, A. Aleksandrov, C. Deibele, and S. Henderson, Pasta-an rf phase scan and tuning application, in Proceedings of the 21st Particle Accelerator Conference, Knoxville, TN, 2005 (IEEE, Piscataway, NJ, 2005), pp. 1491-1493.

[10] P. Harris, H. Bryant, A. Mohagheghi, R. Reeder, C. Tang, J. Donahue, C. Quick, D. Rislove, H. Sharifian, H. Tootoonchi, and T. Altman, Measurement and reduction of momentum spread in the lampf linac beam, Nucl. Instrum. Methods Phys. Res., Sect. A 292, 254 (1990).

[11] D. Holtkamp and C. Quick, A new method of h beamenergy and energy-spread diagnostics, Nucl. Instrum. Methods Phys. Res., Sect. A 287, 348 (1990).

[12] S. Henderson et al., The spallation neutron source accelerator system design, Nucl. Instrum. Methods Phys. Res., Sect. A 763, 610 (2014).

[13] V. Danilov, A. Aleksandrov, S. Assadi, S. Henderson, N. Holtkamp, T. Shea, A. Shishlo, Y. Braiman, Y. Liu, J.
Barhen, and T. Zacharia, Three-step $\mathrm{h}^{-}$charge exchange injection with a narrow-band laser, Phys. Rev. Accel. Beams 6, 053501 (2003).

[14] S. Cousineau, A. Rakhman, M. Kay, A. Aleksandrov, V. Danilov, T. Gorlov, Y. Liu, M. Plum, A. Shishlo, and D. Johnson, First Demonstration of Laser-assisted Charge Exchange for Microsecond Duration $\mathrm{h}^{-}$Beams, Phys. Rev. Lett. 118, 074801 (2017).

[15] S. Cousineau, A. Rakhman, M. Kay, A. Aleksandrov, V. Danilov, T. Gorlov, Y. Liu, C. Long, A. Menshov, M. Plum, A. Shishlo, A. Webster, and D. Johnson, High efficiency laser-assisted $\mathrm{h}^{-}$charge exchange for microsecond duration beams, Phys. Rev. Accel. Beams 20, 120402 (2017).

[16] P. Virtanen et al., SciPy 1.0: Fundamental algorithms for scientific computing in python, Nat. Methods 17, 261 (2020).

[17] T. Gorlov, A. Aleksandrov, S. Cousineau, Y. Liu, A. Rakhman, and A. Shishlo, Sequential excitation scheme for laser stripping for $\mathrm{a} \mathrm{h}^{-}$beam, Phys. Rev. Accel. Beams 22, 121601 (2019).

[18] http://energy.gov/downloads/doe-public-access-plan. 